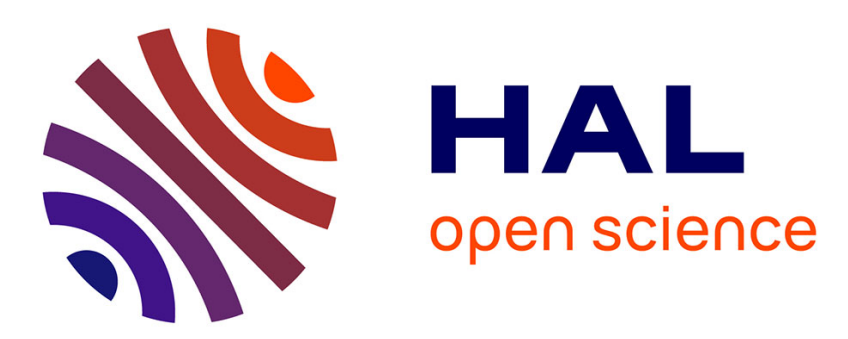

\title{
Stability criterion of spatially inhomogeneous solutions to the Vlasov equation \\ Shun Ogawa
}

\section{To cite this version:}

Shun Ogawa. Stability criterion of spatially inhomogeneous solutions to the Vlasov equation. Journal of Physics A: Mathematical and Theoretical, 2017, 50 (45), pp.455001. 10.1088/1751-8121/aa8dbb . hal-01661095

\section{HAL Id: hal-01661095 \\ https://hal.science/hal-01661095}

Submitted on 18 May 2018

HAL is a multi-disciplinary open access archive for the deposit and dissemination of scientific research documents, whether they are published or not. The documents may come from teaching and research institutions in France or abroad, or from public or private research centers.
L'archive ouverte pluridisciplinaire HAL, est destinée au dépôt et à la diffusion de documents scientifiques de niveau recherche, publiés ou non, émanant des établissements d'enseignement et de recherche français ou étrangers, des laboratoires publics ou privés. 


\title{
Stability criterion of spatially inhomogeneous solutions to Vlasov equation
}

\author{
Shun Ogawa * \\ Aix Marseille Univ., Université de Toulon, CNRS, CPT, Marseille, France \\ RIKEN Brain Science Institute, Hirosawa, Wako, Saitama, 351-0198 Japan
}

\begin{abstract}
Stability of spatially inhomogeneous stationary solutions to the Vlasov equation for multi-dimensional systems is investigated. The formal stability criterion taking into account all of the Casimir invariants is derived in an explicit form without use of the angle-action variables of a single particle's Hamiltonian associated with a stationary solutions to the Vlasov equation, when biorthogonal basis can be defined.
\end{abstract}

\section{Introduction}

Temporal evolution of systems with long range interaction (LRI) is approximately described by the Vlasov equation (also called the collisionless Boltzmann equation) which is an evolution equation of a single particle density function, when the number of particles are large enough [1-5]. This approximation gets to be exact when one takes a large population limit (mean-field limit) [6-8]. This equation is hence a basic tool for analyzing mesoscopic dynamics of Hamiltonian systems with LRI, for example, plasmas [4] and self-gravitating systems [5]. One of remarkable phenomena observed in the LRI systems is existence of long-lasting out-of-equilibrium stationary states called quasistationary states (QSSs) and slowly equilibration in the relaxation process. The QSSs are regarded as stable stationary solutions to the Vlasov equation [9-12] . The LRI system is often trapped in a QSS and its duration diverges as the number $N$ of elements consisting the system. After that, the finite $N$ effect appears and the temporal evolution is no longer described by the Vlasov equation and is described by the kinetic equation such as Balescu-Lenard equation [1-4]. Then, the system leaves the QSS and relaxes to the thermal equilibrium state. Such a relaxation process is directly observed in the $N$ body simulations for the Hamiltonian mean field (HMF) model (globally coupled XY model with inertial terms) [13] in Refs. $[9,14,15]$ and for the $\alpha$-HMF model with $\alpha \leq 1$ (the coupling constant decays as $r^{-\alpha}$ where $r$ denotes distance between lattice points) [16] in Refs. [17, 18]. The duration $\tau_{\mathrm{QSS}}$ of QSS diverges as, for instance, $\tau_{\mathrm{QSS}} \sim N^{v}$ with $v>0$ for the HMF model and the $\alpha$-HMF model with $\alpha<1[9,10,14,15,18], \tau_{\mathrm{QSS}} \sim \ln N$ for the $\alpha$-HMF model with $\alpha=1$ [18], and $\tau_{\mathrm{QSS}} \sim N / \ln N$ for self-gravitating systems [5].

The stability analysis for the Vlasov equation is considered to be a first step of study on QSSs because it clarifies which kind of solutions can be QSSs. For the spatially homogeneous stationary solutions, the simple criteria of formal and spectral stability have been already derived in Refs. [9, 19-22]. It is shown rigorously that some stable homogeneous states are valid for the finite $N$ system within a time scale $\tau_{\text {st }} \sim$ $N^{1 / 8}$ [12]. The asymptotic stability in the nonlinear regime is rigorously shown for the Vlasov-Poisson system in the study on nonlinear Landau damping [23].

The aim of this article is to derive a precise formal stability criterion for spatially inhomogeneous stationary solutions to the Vlasov equation in an explicit form. The stability of inhomogeneous states has been investigated in the context of astrophysics for a long time [5,24-27], and it has been investigated for

*shun.ogawa@riken.jp 
simple systems with long-range interaction $[28,29]$. The nonlinear (orbital) stability is rigorously investigated recently for the spherical gravitating systems [30] and for the HMF model [31].

The spectral stability has been investigated by use of the Nyquist methods [19-22]. In these works the back ground stationary state is assumed to be spatially homogeneous, and an explicit form of the plasmas dispersion function is required. Thus it is difficult to look into stability of spatially inhomogeneous states by using the Nyquist method. There exists another way to derive a stability criterion for spatially inhomogeneous states of the HMF model [28], and the explicit form of dispersion function is necessary in this procedure too, so that it cannot be applied for stationary states whose effective Hamiltonian is non-integrable.

In this article, the formal stability $[9,28,29,32,33]$ is investigated. Let $f_{0}$ be a stationary solution to the Vlasov equation and also be a critical point of some invariant functional (The detail is exhibited in Sec. 3.). A solution $f_{0}$ is said to be formally stable when a second variation ("Hessian") of the invariant functional around $f_{0}$ is positive or negative definite. In this case, $L^{2}$-like-norm of perturbation given by this "Hessian" is an invariant of the linearized Vlasov equation, so that the formally stable solution is linearly stable.

The Vlasov equation has infinitely many constraint conditions coming from the Casimir invariant functionals. Then a physically relevant perturbation satisfies these constraint conditions within a linear order. The problem of dealing these constraints appeared in a stability analysis of perfect fluid flows [33, 34]. More recently, this is a main problem to derive the asymptotic form of the linear response of the Vlasov systems around spatially inhomogeneous states $[14,35,36]$. The QSSs are looked as stable fixed points on a constraint "surface" and these constraints prevent systems relaxing to the equilibrium state [14], and the solution to the linearized Vlasov equation around $f_{0}$ should be on the tangent "plane" at $f_{0}$ in a constraint "surface." When $f_{0}$ is spatially homogeneous [9] or is inhomogeneous whose effective single particle Hamiltonian is integrable [28], the formal stability criterion taking into account all the Casimir constraints is derived. Stationary states with nonintegrable effective Hamiltonians are out of scope in these previous studies. However, for a system in two or three dimensional spaces, the effective Hamiltonians are nonintegrable in general. In Ref. [29], the linear stability is investigated without use of the angle-action variables, and less-refined formal stability criteria taking into account a finite number of invariants have been derived. But the formal stability criterion taking into account all of the Casimir constraints has not been derived, because it requires to determine an infinite number of Lagrangian multipliers associated with the Casimir constraints [29], and it is impossible in general.

Some stationary states are stable against the perturbation violating some Casimir constraints. For instance, if an only normalization condition is taken into account, the inhomogeneous thermal equilibria are judged to be stable in the HMF model [29]. On the other hand, some perturbation violating the constraint conditions can bring about instability. For instance, if one does not take into account any constraint condition, all equilibria in the ordered phase are not judged to be stable in the HMF model [29]. Further, as shown in Ref. [28], some inhomogeneous solutions are not judged to be stable when one use the stability criterion taking into account only normalization of density functions, even though this is formally stable. Thus, it is important in practice to derive the stability criterion taking into account infinitely many Casimir invariants without use of the angle-action variables.

We then derive the formal stability criterion without use of the angle-action variables by expressing the constraint conditions in a simpler manner as in Ref. [36] (a similar formula is found in Ref. [25, 30]). The formal stability criterion is explicitly written in terms of the positive or negative definiteness of a matrix in a similar manner with the previous result [28]. Everything is given a priori in this matrix, so that we can judge the stability of $f_{0}$ by substituting it into the criterion, in principle. When this formula is applied for the one-dimensional (1d) systems, this matrix coincides with the dispersion matrix [5,37] with null frequency. For the multi-dimensional systems, the inverse matrix appears in the linear response and the zero-field susceptibility diverges when the matrix has a null eigenvalue [36]. The marginally formally stable solutions are the marginally spectrally stable ones not only in the $1 \mathrm{~d}$ systems but also in the multidimensional systems.

The rest part of this article is organized as follows: We introduce the Vlasov equation and the constraint conditions of Vlasov dynamics in Sec. 2. In Sec. 3, we exhibit a definition of formal stability and 
derive its criterion. We check the present result includes the previous one for the $1 \mathrm{~d}$ HMF model, and derive the stability criterion for the 2d HMF model. The article is summarized in Sec. 4.

\section{Vlasov equation}

\subsection{Model}

A model dealt in this article is an $N$-body system with Hamiltonian, in the $d$ dimensional real space,

$$
H_{N}=\sum_{i=1}^{N} \frac{\left\|\boldsymbol{p}_{i}\right\|^{2}}{2}+\frac{1}{2 N} \sum_{i, j=1}^{N} V\left(\boldsymbol{q}_{i}-\boldsymbol{q}_{j}\right),
$$

where $\boldsymbol{q}_{i} \in \mathbb{D}^{d} \subset \mathbb{R}^{d}$ and its conjugate momentum $\boldsymbol{p}_{i} \in \mathbb{R}^{d}$, for $i=1,2, \cdots, N$. We assume that the domain $\mathbb{D}^{d}$ is bounded and the potential $V$ is smooth. In the limit $N \rightarrow \infty$, the temporal evolution is well described by the Vlasov equation [6-8],

$$
\begin{aligned}
& \frac{\partial f}{\partial t}+\{\mathscr{H}[f], f\}=0, \quad \mathscr{H}[f]=\frac{\|\boldsymbol{p}\|^{2}}{2}+\mathscr{V}[f](\boldsymbol{q}), \\
& \mathcal{V}[f]=\iint V\left(\boldsymbol{q}-\boldsymbol{q}^{\prime}\right) f\left(\boldsymbol{q}^{\prime}, \boldsymbol{p}^{\prime}, t\right) \mathrm{d} \boldsymbol{q}^{\prime} \mathrm{d} \boldsymbol{p}^{\prime},
\end{aligned}
$$

where $f(\boldsymbol{q}, \boldsymbol{p}, t)$ is a single particle density function, and the Poisson bracket is given by $\{a, b\}=\frac{\partial a}{\partial \boldsymbol{p}} \cdot \frac{\partial b}{\partial \boldsymbol{q}}-$

$\frac{\partial a}{\partial \boldsymbol{q}} \cdot \frac{\partial b}{\partial \boldsymbol{p}}$. The Vlasov equation has infinitely many stationary solutions, and we here deal mainly with a stationary solution $f_{0}$ depending only on the one particle effective Hamiltonian $\mathscr{H}_{0}$,

$$
f_{0}(\boldsymbol{q}, \boldsymbol{p})=F_{0}\left(\mathscr{H}_{0}(\boldsymbol{q}, \boldsymbol{p})\right), \quad \mathscr{H}_{0}(\boldsymbol{q}, \boldsymbol{p})=\mathscr{H}\left[f_{0}\right],
$$

where $F_{0}$ is a monotonically decreasing function. In the similar manner, we can deal with a stationary solution $f_{0}$ depending on two integrals as follows

$$
f_{0}(\boldsymbol{q}, \boldsymbol{p})=F_{0}\left(\mathscr{H}_{0}(\boldsymbol{q}, \boldsymbol{p})+\bar{v} L(\boldsymbol{q}, \boldsymbol{p})\right), \quad \mathscr{H}_{0}(\boldsymbol{q}, \boldsymbol{p})=\mathscr{H}\left[f_{0}\right]
$$

where $L$ is an additional invariant of the Hamiltonian system with $\mathscr{H}_{0}$, and where $F_{0}$ is monotonic with respect to $\mathscr{H}_{0}+\bar{v} L$ where $\bar{v}$ is a constant. The stationary solution $f_{0}$ is assumed to depend on a linear combination of two integrals because we focus on the stationary solution $f_{0}$ which is also a solution to the optimization problem with some constraint conditions in the present article.

We additionally assume that biorthogonal basis can be defined in this model, and this is introduced in a forthcoming section.

\subsection{Casimir invariants}

The Vlasov equation has infinitely many constraint conditions coming from Casimir functionals,

$$
\mathscr{C}[f]=\iint c(f(\boldsymbol{q}, \boldsymbol{p}, t)) \mathrm{d} \boldsymbol{q} \mathrm{d} \boldsymbol{p},
$$

which are conserved in the temporal evolution for any smooth function $c$. Then solutions to the linearized Vlasov equation around $f_{0}$ should be on the tangent "plane" at $f_{0}$ on the iso-Casimirs "surface," so that the physically relevant perturbation $\delta f$ satisfies, within a linear order,

$$
\iint c^{\prime}\left(f_{0}(\boldsymbol{q}, \boldsymbol{p})\right) \delta f(\boldsymbol{q}, \boldsymbol{p}) \mathrm{d} \boldsymbol{q} \mathrm{d} \boldsymbol{p}=0
$$


for any $c$, where $c^{\prime}(x)=\mathrm{d} c / \mathrm{d} x$. Since $f_{0}=F_{0}\left(\mathscr{H}_{0}\right)$ is assumed to be monotonic with respect to $\mathscr{H}_{0}$, it is shown that this constraint condition is equivalent to the condition:

$$
\iint R\left(\mathscr{H}_{0}(\boldsymbol{q}, \boldsymbol{p})\right) \delta f(\boldsymbol{q}, \boldsymbol{p}) \mathrm{d} \boldsymbol{q} \mathrm{d} \boldsymbol{p}=0
$$

for any function $R$ [28]. This is equivalent to the condition:

$$
\iint R\left(\mathscr{H}_{0}(\boldsymbol{q}, \boldsymbol{p})\right)\langle\delta f(\boldsymbol{q}, \boldsymbol{p})\rangle_{\mathscr{H}_{0}} \mathrm{~d} \boldsymbol{q} \mathrm{d} \boldsymbol{p}=0
$$

(see A for the derivation). The constraint condition are expressed as follows:

$$
\langle\delta f\rangle_{\mathscr{H}_{0}}=0,
$$

for almost every $\mathscr{H}_{0}$, where $\langle\bullet\rangle_{\mathscr{\ell}_{0}}$ denotes the average over the iso- $\mathscr{H}_{0}$ set. Thus, the accessible perturbation can be written as

$$
\delta f(\boldsymbol{q}, \boldsymbol{p})=g(\boldsymbol{q}, \boldsymbol{p})-\langle g\rangle_{\mathcal{H}_{0}(\boldsymbol{q}, \boldsymbol{p})} .
$$

It should be remarked that the condition (6)-(9) is not for the initial state of the perturbation $\delta f(t=$ 0 ) but for the relevant dynamical part of the perturbation whose constrained on a constraint surface. Even if the $\delta f(t=0)$ does not satisfies the constraint condition (9), the linearized dynamics should be constrained on a tangent plane of the constraint surface and the directions that are not parallel to the tangent plane are not accessible. Thus, it is worthwhile to check the formal stability to show the linear stability of the stationary solution $f_{0}$.

When the system has an additional invariant $L$, we should find a condition likes Eq. (9) for each steady state. If the stationary solution $f_{0}$ depends on only $\mathscr{H}_{0}$, the physically relevant perturbation satisfies Eq. (9). When $f_{0}$ is written as Eq. (4), the accessible perturbation satisfies

$$
\langle\delta f\rangle_{\mathcal{H}_{0}+\bar{v} L}=0 .
$$

Then the stability criterion for $f_{0}$ in Eq. (4) is derived as done for the stationary solution depending only $\mathscr{H}_{0}$ by replacing $\mathscr{H}_{0}$ with $\mathscr{H}_{0}+\bar{v} L$.

\section{Formal stability}

\subsection{Definition of formal stability}

The concept of the formal stability [32] is introduced here. Let us consider the stationary solution $f_{0}$ to the Vlasov equation, which is a solution to an optimization problem with constraints, normalization and energy conservation;

$$
\begin{aligned}
\text { maximizing } \mathscr{S}[f] & =\iint s(f) \mathrm{d} \boldsymbol{q} \mathrm{d} \boldsymbol{p}, \\
\text { subject to } \mathscr{N}[f] & =\iint f \mathrm{~d} \boldsymbol{q} \mathrm{d} \boldsymbol{p}=1, \\
\mathscr{E}[f] & =\iint \frac{\|\boldsymbol{p}\|^{2}}{2} f \mathrm{~d} \boldsymbol{q} \mathrm{d} \boldsymbol{p}+\frac{1}{2} \iint \mathcal{V}[f] f \mathrm{~d} \boldsymbol{q} \mathrm{d} \boldsymbol{p}=E,
\end{aligned}
$$

where $s$ is a strictly concave function. This is similar to the maximizing entropy principle. A solution to the optimization problem (12) is

$$
f_{0}=\left(s^{\prime}\right)^{-1}\left(\beta \mathscr{H}_{0}+\alpha\right),
$$

where $s^{\prime}(x)=\mathrm{d} s / \mathrm{d} x$, and $\alpha$ and $\beta$ are Lagrangian multipliers corresponding to the normalization and the energy conservation respectively. Since $s$ is concave, $f_{0}$ satisfies the condition (3) when $\beta>0$. Taking the second variation of the functional $\mathscr{F}=\mathscr{S}-\alpha \mathscr{N}-\beta \mathscr{E}$ around $f_{0}$, we have

$$
\delta^{2} \mathscr{F}\left[f_{0}\right]\left[\delta f, \delta f^{*}\right]=\beta \iint\left(\frac{|\delta f|^{2}}{\gamma\left(\mathscr{C}_{0}\right)}-\delta f^{*} \mathcal{V}[\delta f]\right) \mathrm{d} \boldsymbol{q} \mathrm{d} \boldsymbol{p},
$$


where $z^{*}$ denotes complex conjugate of $z \in \mathbb{C}$ and $\gamma\left(\mathscr{H}_{0}\right)=\beta / s^{\prime \prime}\left(f_{0}\right)=\mathrm{d} f_{0} / \mathrm{d} \mathscr{H}_{0}$. The functional (14) is an invariant of the linearized Vlasov dynamics. When the quadratic form (14) is negative for any perturbations $\delta f$ satisfying Eq. (9), the stationary solution $f_{0}$ is said to be formally stable. The formally stable solution is linearly stable [32].

Let us consider the case that there is an additional invariant,

$$
\mathscr{L}[f]=\iint L(\boldsymbol{q}, \boldsymbol{p}) f \mathrm{~d} \boldsymbol{q} \mathrm{d} \boldsymbol{p}=\bar{L} .
$$

The solution to the optimization problem is $f_{0}=\left(s^{\prime}\right)^{-1}(\beta \Psi+\alpha)$, where $\Psi=\mathscr{H}_{0}+v L / \beta$ and $v$ is a Lagrangian multiplier associated with the condition (15). The second variation of the functional (15) is zero, so that it does not appear in Eq. (14). Then, we just put $\gamma\left(\mathscr{H}_{0}, L\right)=\mathrm{d} f_{0} / \mathrm{d} \Psi$ in Eq. (14) instead of $\gamma\left(\mathscr{H}_{0}\right)$.

\subsection{Bi-orthogonal basis}

A useful tool for analyzing perturbations of the Vlasov systems are biorthogonal basis, $\left\{d_{i}(\boldsymbol{q})\right\}_{i \in \rrbracket}$ and

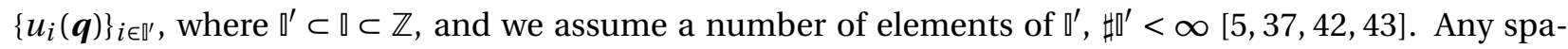
tial density perturbations $\delta \rho(\boldsymbol{q})=\int \delta f(\boldsymbol{q}, \boldsymbol{p}) \mathrm{d} \boldsymbol{p}$ is spanned with $\left\{d_{i}(\boldsymbol{q})\right\}_{i \in \rrbracket}$, and $\left\{u_{i}(\boldsymbol{q})\right\}_{i \in \mathbb{\rrbracket}^{\prime}}$ are given by

$$
u_{i}(\boldsymbol{q})=\left(V * d_{i}\right)(\boldsymbol{q}) \equiv \int V\left(\boldsymbol{q}-\boldsymbol{q}^{\prime}\right) d_{j}\left(\boldsymbol{q}^{\prime}\right) \mathrm{d} \boldsymbol{q}^{\prime} .
$$

They are supposed to satisfy

$$
\int u_{i}^{*}(\boldsymbol{q}) d_{j}(\boldsymbol{q}) \mathrm{d} \boldsymbol{q}=\lambda_{i} \delta_{i j}
$$

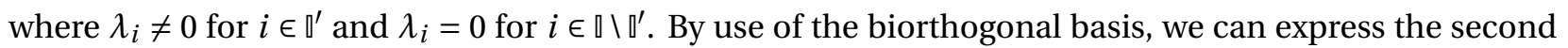
term of right hand side in Eq. (14) as

$$
\iint \delta f^{*} \mathcal{V}[\delta f] \mathrm{d} \boldsymbol{q} \mathrm{d} \boldsymbol{p}=\sum_{i \in \mathbb{\|}^{\prime}} \frac{1}{\lambda_{i}}\left|\iint u_{i}^{*} \delta f \mathrm{~d} \boldsymbol{q} \mathrm{d} \boldsymbol{p}\right|^{2} .
$$

The derivation is exhibited in $\mathrm{B}$.

\subsection{Formal stability criterion}

Let us derive the formal stability criterion explicitly. We suppose that the single particle Hamiltonian $\mathscr{H}_{0}$ has only one integral, $\mathscr{H}_{0}$ per se. Substituting Eq. (10) into Eq. (14), we express the formal stability condition as

$$
\mathscr{G}\left[g, g^{*}\right]=\delta^{2} \mathscr{F}\left[f_{0}\right]\left[g-\langle g\rangle_{\mathscr{C}_{0}}, g^{*}-\left\langle g^{*}\right\rangle_{\mathscr{H}_{0}}\right] / \beta<0,
$$

for any $g$. Our strategy is to find a $g_{\max }$ maximizing $\mathscr{G}$ under a constraint,

$$
\mathscr{M}\left[g, g^{*}\right]=\sum_{i \in \mathbb{D}^{\prime}}\left|\iint u_{i}^{*}\left(g-\langle g\rangle_{\mathscr{H}_{0}}\right) \mathrm{d} \boldsymbol{q} \mathrm{d} \boldsymbol{p}\right|^{2}=1,
$$

and to derive the condition so that $\mathscr{G}\left[g_{\max }, g_{\max }^{*}\right]<0$ which is equivalent to Eq. (19). The value 1 itself in the right hand side of Eq. (20) is not important. The condition $\mathscr{M}\left[g, g^{*}\right]>0$ is really important. This is because the rescaling $g \mapsto c g$ with a constant $c$ just changes the values of functionals as $\mathscr{G} \mapsto|c|^{2} \mathscr{G}$ and $\mathscr{M} \mapsto|c|^{2} \mathscr{M}$ and it does not affect the sign of $\mathscr{G}$. When $\mathscr{M}\left[g, g^{*}\right]=0$, then $\mathscr{G}\left[g, g^{*}\right]<0$ because $\gamma\left(\mathscr{H}_{0}\right)<0$. Thus such a perturbation does not bring about instability. By using a vector $\boldsymbol{\xi}=\left(\xi_{i}\right)_{i \in \mathbb{l}^{\prime}}$ given by

$$
\xi_{i}[g]=\iint u_{i}^{*}\left(g-\langle g\rangle_{\mathscr{H}_{0}}\right) \mathrm{d} \boldsymbol{q} \mathrm{d} \boldsymbol{p},
$$


the constraint (20) is expressed as $\|\boldsymbol{\xi}\|=1$, where $\|\bullet\|$ denotes Euclidean norm. We write $\xi_{i}\left[g_{\max }\right]=\bar{\xi}_{i}$ and $\boldsymbol{\xi}\left[g_{\max }\right]=\overline{\boldsymbol{\xi}}$. Let $\hat{A}^{\dagger}$ denote the Hermitian conjugate of a matrix $\hat{A}$ and $\boldsymbol{a}^{\dagger}=\left(\boldsymbol{a}^{*}\right)^{\mathrm{T}}$ where the superscript T denotes transposition. By use of the equality (see A for the derivation),

$$
\iint a\langle b\rangle_{\mathscr{H}_{0}} \mathrm{~d} \boldsymbol{q} \mathrm{d} \boldsymbol{p}=\iint\langle a\rangle_{\mathscr{H}_{0}} b \mathrm{~d} \boldsymbol{q} \mathrm{d} \boldsymbol{p},
$$

for any functions $a$ and $b$ for which the integrals are defined, we have $g_{\max }$ holding $\delta \mathscr{G} / \delta g-\eta \delta \mathscr{M} / \delta g=0$ as

$$
g_{\max }^{*}-\left\langle g_{\max }^{*}\right\rangle_{\mathscr{H}_{0}}=\gamma\left(\mathscr{H}_{0}\right) \sum_{i \in \rrbracket^{\prime}}\left(\frac{1}{\lambda_{i}}+\eta\right) \bar{\xi}_{i}^{*}\left(u_{i}^{*}-\left\langle u_{i}^{*}\right\rangle_{\mathscr{H}_{0}}\right),
$$

where $\eta$ is a Lagrangian multiplier with respect to the constraint condition (20). Let $\zeta_{i}=\left(\lambda_{i}^{-1}+\eta\right) \bar{\xi}_{i}$ and $\zeta=\left(\zeta_{i}\right)_{i \in \mathbb{I}^{\prime}}$. Substituting Eq. (23) into Eq. (19), we have the condition of the formal stability of $f_{0}$ as

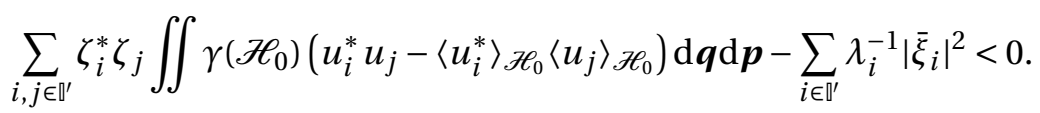

This inequality is similar to Eq. (1.26) in Ref. [30]. For simplicity, we introduce several notations;

$$
F_{i j}=\iint \gamma\left(\mathscr{H}_{0}\right)\left(u_{i}^{*} u_{j}-\left\langle u_{i}^{*}\right\rangle_{\mathscr{H}_{0}}\left\langle u_{j}\right\rangle_{\mathscr{H}_{0}}\right) \mathrm{d} \boldsymbol{q} \mathrm{d} \boldsymbol{p},
$$

$\hat{F}=\left(F_{i j}\right)_{(i, j) \in \mathbb{\|}^{\prime} \times \mathbb{1}^{\prime}}$, and $\hat{\Lambda}=\operatorname{diag}\left(\lambda_{1}, \lambda_{2}, \cdots, \lambda_{\sharp \mathbb{D}^{\prime}}\right)$. By use of them, a relation $\overline{\boldsymbol{\xi}}=\hat{F} \boldsymbol{\zeta}$ is derived from Eq. (23). We thus have the formal stability condition as $\zeta^{\dagger} \hat{F}\left(\hat{1}-\hat{\Lambda}^{-1} \hat{F}\right) \zeta<0$, where $\hat{1} \in \mathbb{C}^{\sharp 0^{\prime} \times \sharp 0^{\prime}}$ is the unit matrix and we have used the fact $\hat{F}=\hat{F}^{\dagger}$. The matrix $\hat{F}$ is negative definite, because

$$
F_{i j}=\iint \gamma\left(\mathscr{H}_{0}\right)\left(\left\langle u_{i}^{*} u_{j}\right\rangle_{\mathscr{O}_{0}}-\left\langle u_{i}^{*}\right\rangle_{\mathscr{H}_{0}}\left\langle u_{j}\right\rangle_{\mathscr{H}_{0}}\right) \mathrm{d} \boldsymbol{q} \mathrm{d} \boldsymbol{p}
$$

the matrix $\left(\left\langle u_{i}^{*} u_{j}\right\rangle_{\mathscr{C}_{0}}-\left\langle u_{i}^{*}\right\rangle_{\mathscr{H}_{0}}\left\langle u_{j}\right\rangle_{\mathscr{H}_{0}}\right)_{(i, j) \in \mathbb{1}^{\prime} \times \mathbb{1}^{\prime}}$ is positive definite, and $\gamma\left(\mathscr{H}_{0}\right)<0$. It is therefore shown the main claim of this article.

Main Claim Let $f_{0}=F_{0}\left(\mathscr{H}_{0}\right)\left(F_{0}^{\prime}\left(\mathscr{H}_{0}\right)<0\right)$ be a stationary solution to the Vlasov equation and $\mathscr{H}_{0}$ have only one integral of motion, $\mathscr{H}_{0}$ itself. A stationary solution $f_{0}$ to the Vlasov equation is formally stable if and only if the matrix $\hat{D}\left[f_{0}\right]=\hat{1}-\hat{\Lambda}^{-1} \hat{F}$ is positive definite.

It should be remarked that the matrix $\hat{D}\left[f_{0}\right]$ is the matrix form of dispersion functions with null frequency when the spatial dimension $d=1[5,37,42]$. Further, the inverse matrix of $\hat{D}\left[f_{0}\right]$ appears in a linear response formula for the $1 \mathrm{~d}$ and multi-dimensional systems $[36,38]$. Thus, the zero minimum eigenvalue of $\hat{D}\left[f_{0}\right]$ might also correspond to the marginal spectral stability, because the zero field susceptibility diverges when $\operatorname{det} \hat{D}\left[f_{0}\right]=0$.

We mention the relation between the present main claim and the classical results on the spherical stellar system. The Antonov's variational principle [5] says: For the stellar system in the state $f_{0}=F_{0}\left(\mathscr{H}_{0}\right)$ $\left(F_{0}^{\prime}<0\right)$ is stable if and only if

$$
\mathscr{W}_{\mathrm{A}}\left[f_{0}\right][h] \equiv-\delta^{2} \mathscr{F}\left[\left\{h, f_{0}\right\},\left\{h, f_{0}\right\}\right] \beta>0,
$$

for any $h(\boldsymbol{q}, \boldsymbol{p})$. Furthermore, it is shown that $F_{0}\left(\mathscr{H}_{0}\right)$ with $F_{0}^{\prime}<0$ is stable by showing $\mathscr{W}_{\mathrm{A}}\left[f_{0}\right][h]>0[5]$. We call this statement the classical statement. Let us recall that $f_{0}=F_{0}\left(\mathscr{H}_{0}\right)\left(F_{0}^{\prime}<0\right)$ is formally stable if and only if (Eq. (19))

$$
-\mathscr{G}\left[g, g^{*}\right]=-\delta^{2} \mathscr{F}\left[g-\langle g\rangle_{\mathcal{H}_{0}}, g^{*}-\left\langle g^{*}\right\rangle_{\mathscr{H}_{0}}\right] / \beta>0 .
$$

This is similar to Eq. (27). Let $\mathbb{W}$ and $\mathbb{G}$ be sets of $\delta f$ such that

$$
\begin{array}{ll}
\exists h(\boldsymbol{q}, \boldsymbol{p}), & \text { s.t. } \delta f=\left\{h, f_{0}\right\}, \\
\exists g(\boldsymbol{q}, \boldsymbol{p}), & \text { s.t. } \delta f=g-\langle g\rangle_{\mathscr{H}_{0}},
\end{array}
$$


respectively. Since $\left\langle\left\{h, f_{0}\right\}\right\rangle_{\mathscr{H}_{0}}=0$, we have $\mathbb{W} \subset \mathbb{G}$. It is hence shown that $W_{\mathrm{A}}\left[f_{0}\right][h]>0$ for any $h$ and Antonov's variational principle holds true, when Eq. (19) holds, or equivalently, $\hat{D}\left[f_{0}\right]$ is positive definite. If there exists $h$ satisfying

$$
F_{0}^{\prime}\left(\mathscr{H}_{0}\right)\left\{h, \mathscr{H}_{0}\right\}=g-\langle g\rangle_{\mathscr{H}_{0}},
$$

for each $g$, we have $\mathbb{W}=\mathbb{G}$. Thus, the positive definiteness of $\hat{D}\left[f_{0}\right]$ is equivalent to the condition (27) and we have: For the spherical stellar system, if $F_{0}\left(\mathscr{H}_{0}\right)$ satisfies $F_{0}^{\prime}\left(\mathscr{H}_{0}\right)<0$ for all $\mathscr{H}_{0}$, then $\hat{D}\left[f_{0}\right]$ is positive definite. On the other hand, $F_{0}^{\prime}\left(\mathscr{H}_{0}\right)<0$ is assumed in our main claim, so that the present criterion is not so powerful for the spherical stellar systems, when $\mathbb{W}=\mathbb{G}$.

The set $\mathbb{D}^{\prime}$ is assumed to be a finite set in the present section. One problem is that the matrix $\hat{D}\left[f_{0}\right]$ becomes infinite matrix, $i$. e. $\mathbb{\nabla}^{\prime}$ is not a finite set in general. Thus, some truncation or approximation of the model $V\left(\boldsymbol{q}-\boldsymbol{q}^{\prime}\right)=\sum_{i \in \mathbb{1}^{\prime}} u_{i}(\boldsymbol{q}) u_{i}^{*}\left(\boldsymbol{q}^{\prime}\right)$ is necessary. When the biorthogonal basis are the Fourier modes, and $V$ is smooth enough, it may be possible to truncate the matrix elements $D\left[f_{0}\right]_{i j}$ for large $i$ or $j$ when one computes the determinant. We do not deal with this problem in this article. In the precedence subsections, two examples are exhibited, in which $\sharp D^{\prime}<\infty$, the $1 \mathrm{~d}$ and two dimensional (2d) HMF models $[13,39,40]$. Both of them are out of scope of the classical statement. The $2 \mathrm{~d}$ one is out of scope of the previous paper [28], because the angle-action variables are not constructed.

\subsection{One-dimensional Hamiltonian mean-field model}

Let us examine the obtained criterion includes the one obtained in the previous article [28], the formal stability criterion for the HMF model whose Hamiltonian is

$$
H=\sum_{i=1}^{N} \frac{p_{i}^{2}}{2}-\frac{1}{2 N} \sum_{i \neq j} \cos \left(q_{i}-q_{j}\right)-h \sum_{i=1}^{N} \cos q_{i},
$$

where $h$ is an external field, $p_{i} \in \mathbb{R}, q_{i} \in[-\pi, \pi)$ for $i=1,2, \cdots, N$. The effective Hamiltonian $\mathscr{H}_{0}$ of the stationary state $f_{0}=F_{0}\left(\mathscr{H}_{0}\right)$ is

$$
\mathscr{H}_{0}=\frac{p^{2}}{2}-\iint \cos \left(q-q^{\prime}\right) f_{0}\left(q^{\prime}, p^{\prime}\right) \mathrm{d} q^{\prime} \mathrm{d} p^{\prime}-h \cos q .
$$

In this case, $\square=\mathbb{N}, \rrbracket^{\prime}=\{1,2\}$ and $d_{i}$ and $u_{i}$ are given respectively by $d_{2 k}=\pi^{-1} \cos (k q), d_{2 k-1}=\pi^{-1} \sin (k q)$, $u_{1}=-\sin q$, and $u_{2}=-\cos q$, and $\lambda_{1}=\lambda_{2}=-1$. Substituting them into the general form (26) of the matrix $\hat{D}=\left(D_{i j}\right)_{(i, j) \in\{1,2\}^{2}}$, we have $D_{12}=D_{21}=0[41]$, and

$$
\begin{aligned}
& D_{11}=1+\iint \frac{d F_{0}\left(\mathscr{H}_{0}\right)}{d \mathscr{H}_{0}}\left(\sin ^{2} q-\langle\sin q\rangle_{\mathscr{H}_{0}(q, p)}^{2}\right) \mathrm{d} q \mathrm{~d} p, \\
& D_{22}=1+\iint \frac{d F_{0}\left(\mathscr{H}_{0}\right)}{d \mathscr{H}_{0}}\left(\cos ^{2} q-\langle\cos q\rangle_{\mathscr{H}_{0}(q, p)}^{2}\right) \mathrm{d} q \mathrm{~d} p .
\end{aligned}
$$

The stationary solution $f_{0}$ is formally stable if and only if $D_{11}>0$ and $D_{22}>0$. Using the angle-action variables, one shows that these coincide with the dispersion function (also called a dielectric function in the context of plasma physics) with null frequency. This criterion is hence the same with the one obtained in the previous study [28], and with the criterion of the orbital stability [31].

\subsection{Two-dimensional Hamiltonian mean-field model}

The 2d HMF model is out of scope of the previous study [28], because it is impossible to construct the angle-action variables for the effective Hamiltonian of the inhomogeneous stationary states. The $N$-body Hamiltonian of this model is

$$
\begin{aligned}
H= & \sum_{i=1}^{N} \frac{\left\|\boldsymbol{p}_{i}\right\|^{2}}{2}-h_{x} \sum_{i=1}^{N} \cos x_{i}-h_{y} \sum_{i=1}^{N} \cos y_{i} \\
& -\frac{1}{2 N} \sum_{i \neq j}\left[\cos \left(x_{i}-x_{j}\right)+\cos \left(y_{i}-y_{j}\right)+\cos \left(x_{i}-x_{j}\right) \cos \left(y_{i}-y_{j}\right)\right],
\end{aligned}
$$


where $\boldsymbol{p}_{i}=\left(p_{x i}, p_{y i}\right) \in \mathbb{R}^{2}$ and $\boldsymbol{q}_{i}=\left(x_{i}, y_{i}\right) \in[-\pi, \pi)^{2}$ for $i=1,2, \cdots, N[39,40]$. The effective Hamiltonian for the stationary state with the external field $h_{x}=h_{y}=h$ is

$$
\begin{aligned}
\mathscr{H}_{0} & =\frac{p_{x}^{2}+p_{y}^{2}}{2}+\mathcal{V}(x, y), \\
\mathcal{V}(x, y) & =-(M+h) \cos x-(M+h) \cos y-P_{\operatorname{cc}} \cos x \cos y,
\end{aligned}
$$

where we assume that the stationary state $f_{0}(\boldsymbol{q}, \boldsymbol{p})=F_{0}\left(\mathscr{H}_{0}\right)$ is symmetric with respect to both $x$ and $y$, and we define the order parameters in the stationary state $f_{0}$ as $M=\iint \cos x f_{0} \mathrm{~d} \boldsymbol{q} \mathrm{d} \boldsymbol{p}=\iint \cos y f_{0} \mathrm{~d} \boldsymbol{q} \mathrm{d} \boldsymbol{p}$ and $P_{\mathrm{cc}}=\iint \cos x \cos y f_{0} \mathrm{~d} \boldsymbol{q} \mathrm{d} \boldsymbol{p}$. We chose $\left\{u_{i}\right\}_{i \in\{1,2, \cdots, 8\}}$ as

$$
\begin{array}{ccc}
u_{1}=\cos x, & u_{2}=\cos y, & u_{3}=\cos x \cos y, \\
& u_{4}=\sin x, & u_{5}=\sin x \cos y, \\
u_{6}=\sin y, & u_{7}=\cos x \sin y, \\
& u_{8}=\sin x \sin y .
\end{array}
$$

For the symmetric potential $\mathcal{V}$, we have $\left\langle u_{i}\right\rangle_{\mathcal{H}_{0}}=0$, for $i=4,5, \cdots, 8$, and $F_{i j}=0$ when $u_{i} u_{j}$ is odd with respect to $x$ or $y$. Let $0_{m, n}$ be an $m \times n$ null matrix. In the similar manner in Ref. [36], we explicitly write the matrix $\hat{D}=\left(D_{i j}\right)_{(i, j) \in\{1,2, \cdots, 8\}^{2}}$ as

$$
\hat{D}=\left(\begin{array}{cccc}
\hat{D}_{\mathrm{e}} & 0_{3,4} & 0_{3,1} \\
& \hat{D}_{\mathrm{o}} & 0_{2,2} & 0_{4,1} \\
0_{4,3} & 0_{2,2} & \hat{D}_{\mathrm{o}} & \\
0_{1,3} & 0_{1,4} & D_{88}
\end{array}\right)=\operatorname{diag}\left(\hat{D}_{\mathrm{e}}, \hat{D}_{\mathrm{o}}, \hat{D}_{\mathrm{o}}, D_{88}\right),
$$

where $\hat{D}_{\mathrm{e}} \in \mathbb{R}^{3 \times 3}, \hat{D}_{\mathrm{o}} \in \mathbb{R}^{2 \times 2}$, and $D_{88}$ are respectively

$$
\begin{aligned}
\hat{D}_{\mathrm{e}} & =\left(\begin{array}{ccc}
1+G_{1}^{\mathrm{e}} & G_{2}^{\mathrm{e}} & G_{3}^{\mathrm{e}} \\
G_{2}^{\mathrm{e}} & 1+G_{1}^{\mathrm{e}} & G_{3}^{\mathrm{e}} \\
G_{3}^{\mathrm{e}} & G_{3}^{\mathrm{e}} & 1+G_{4}^{\mathrm{e}}
\end{array}\right), \\
\hat{D}_{\mathrm{o}} & =\left(\begin{array}{cc}
1+G_{1}^{\mathrm{o}} & G_{2}^{\mathrm{o}} \\
G_{2}^{\mathrm{o}} & 1+G_{3}^{\mathrm{o}}
\end{array}\right), \\
D_{88} & =1+\iint \frac{d F_{0}\left(\mathscr{\ell}_{0}\right)}{d \mathscr{\ell}_{0}} \sin ^{2} x \sin ^{2} y \mathrm{~d} \boldsymbol{q} \mathrm{d} \boldsymbol{p},
\end{aligned}
$$

and where

$$
\begin{aligned}
G_{1}^{\mathrm{e}} & =\iint \frac{d F_{0}\left(\mathscr{H}_{0}\right)}{d \mathscr{H}_{0}}\left(\cos ^{2} x-\langle\cos x\rangle_{\mathscr{H}_{0}}^{2}\right) \mathrm{d} \boldsymbol{q} \mathrm{d} \boldsymbol{p}, \\
G_{2}^{\mathrm{e}} & =\iint \frac{d F_{0}\left(\mathscr{\ell}_{0}\right)}{d \mathscr{H}_{0}}\left(\cos x \cos y-\langle\cos x\rangle_{\mathscr{H}_{0}}^{2}\right) \mathrm{d} \boldsymbol{q} \mathrm{d} \boldsymbol{p}, \\
G_{3}^{\mathrm{e}} & =\iint \frac{d F_{0}\left(\mathscr{H}_{0}\right)}{d \mathscr{H}_{0}}\left(\cos ^{2} x \cos y-\langle\cos x\rangle_{\mathscr{H}_{0}}\langle\cos x \cos y\rangle_{\mathscr{\ell}_{0}}\right) \mathrm{d} \boldsymbol{q} \mathrm{d} \boldsymbol{p}, \\
G_{4}^{\mathrm{e}} & =\iint \frac{d F_{0}\left(\mathscr{H}_{0}\right)}{d \mathscr{H}_{0}}\left(\cos ^{2} x \cos ^{2} y-\langle\cos x \cos y\rangle_{\mathscr{H}_{0}}^{2}\right) \mathrm{d} \boldsymbol{q} \mathrm{d} \boldsymbol{p},
\end{aligned}
$$

(see Appendix of Ref. [36] for a way to compute numerically them.) and

$$
\begin{aligned}
G_{1}^{\mathrm{o}} & =\iint \frac{d F_{0}\left(\mathscr{H}_{0}\right)}{d \mathscr{H}_{0}} \sin ^{2} x \mathrm{~d} \boldsymbol{q} \mathrm{d} \boldsymbol{p}, \\
G_{2}^{\mathrm{o}} & =\iint \frac{d F_{0}\left(\mathscr{H}_{0}\right)}{d \mathscr{H}_{0}} \sin ^{2} x \cos y \mathrm{~d} \boldsymbol{q} \mathrm{d} \boldsymbol{p}, \\
G_{3}^{\mathrm{o}} & =\iint \frac{d F_{0}\left(\mathscr{H}_{0}\right)}{d \mathscr{H}_{0}} \sin ^{2} x \cos ^{2} y \mathrm{~d} \boldsymbol{q} \mathrm{d} \boldsymbol{p},
\end{aligned}
$$


Thus, for the 2d HMF model, $F_{0}$ is formally stable if and only if $\hat{D}_{\mathrm{e}}$ and $\hat{D}_{\mathrm{o}}$ is positive definite and $D_{88}>0$. The matrix $\hat{D}_{\mathrm{e}}$ appears in the linear response formula [36] and if one of its eigenvalues is 0 , the response diverges. Thus, the marginally formally stable solution $f_{0}$ is the marginally spectrally stable solution.

\section{Summary}

The formal stability criterion of stationary solutions to the Vlasov equation for systems in multi-dimensional space is derived in an explicit form, where the biorthogonal basis can be defined. This criterion takes into account all of the Casimir invariants.

The practical usefulness of the refined criterion has been already mentioned in the previous paper [28]. The less refined criteria taking into account on the finite numbers of invariants cannot be used when the stationary state is close to the marginal stable one with spatial inhomogeneity. Furthermore, we overcome a weak point of the result obtained in Ref. [28]; the criteria has been derived only for the HMF model or its generalized ones such as $\alpha$-HMF model $[16,17]$. We have checked that our general result includes what is obtained in the previous study for the HMF model [28], and the matrix $\hat{D}\left[f_{0}\right]$ coincides with the dispersion matrix when the system is in $1 \mathrm{~d}$ real space.

For the $1 \mathrm{~d}$ systems the minimum eigenvalue of $\hat{D}\left[f_{0}\right]$ is 0 if and only if the operator $\hat{L}\left[f_{0}\right]$ in the linearized Vlasov equation $\partial_{t} \delta f=\hat{L}\left[f_{0}\right][\delta f]$ around $f_{0}$ has an (embedded) eigenvalue 0 . Thus, the marginally formally stable solution $f_{0}$ is also the marginally spectrally stable solution. In the multi-dimensional systems, although the dispersion relation for the non-zero frequency has not been derived, we can obtain the same statement as follows: The matrix $\hat{D}\left[f_{0}\right]^{-1}$ appears in the linear response formula [36]. Thus, the linear response diverges when $\hat{D}\left[f_{0}\right]$ has a null eigenvalue, and the marginally formally stable $f_{0}$ is the marginally spectrally stable. It should be noted that the spectral stability criterion is not obtained and this is an open problem. It should be noted that when the matrix $\hat{D}\left[f_{0}\right]$ is not positive definite, and has both positive and negative eigenvalues, it is possible that a growing (unstable) mode exists around $f_{0}$.

Acknowledgments The author acknowledges the financial support of the A*MIDEX project ( $n^{\circ}$ ANR-11IDEX-0001-02) funded by the "investissements d'Avenir" French Government program, managed by the French National Research Agency (ANR).

\section{A Derivation of Eq. (22)}

We exhibit a derivation of Eq. (22). $\langle X\rangle_{\mathscr{H}_{0}}$ is explicitly written as

$$
\langle X\rangle_{\mathscr{H}_{0}}=\frac{1}{S\left(\mathscr{H}_{0}\right)} \iint X(\boldsymbol{q}, \boldsymbol{p}) \delta\left(\mathscr{H}_{0}-\mathscr{H}_{0}(\boldsymbol{q}, \boldsymbol{p})\right) \mathrm{d} \boldsymbol{q} \mathrm{d} \boldsymbol{p},
$$

where $S$ denotes a measure of iso- $\mathscr{H}_{0}$ in the $\mu$-space, the phase space of single particle,

$$
S(E)=\iint \delta\left(E-\mathscr{H}_{0}(\boldsymbol{q}, \boldsymbol{p})\right) \mathrm{d} \boldsymbol{q} \mathrm{d} \boldsymbol{p} .
$$

We assume that $\left\|\nabla \mathscr{H}_{0}\right\| \neq 0$, so that the iso- $\mathscr{H}_{0}$ sets are null sets in $2 d$-dimensional $\mu$ space for each level. Hereafter, for any function $X(\boldsymbol{q}, \boldsymbol{p}), X, X^{\prime}$, and $X^{\prime \prime}$ denote $X(\boldsymbol{q}, \boldsymbol{p}), X\left(\boldsymbol{q}^{\prime}, \boldsymbol{p}^{\prime}\right)$, and $X\left(\boldsymbol{q}^{\prime \prime}, \boldsymbol{p}^{\prime \prime}\right)$ respectively. The primes do not mean derivatives hereafter. The equation (22) is shown as follows: The right hand side in Eq. (22) is equal to

$$
\iint a\left[\iint \frac{b^{\prime} \delta\left(\mathscr{H}_{0}-\mathscr{H}_{0}^{\prime}\right)}{S\left(\mathscr{H}_{0}\right)} \mathrm{d} \boldsymbol{q}^{\prime} \mathrm{d} \boldsymbol{p}^{\prime}\right] \mathrm{d} \boldsymbol{q} \mathrm{d} \boldsymbol{p}=\iint b^{\prime}\left[\iint \frac{a \delta\left(\mathscr{H}_{0}-\mathscr{H}_{0}^{\prime}\right)}{S\left(\mathscr{C}_{0}^{\prime}\right)} \mathrm{d} \boldsymbol{q} \mathrm{d} \boldsymbol{p}\right] \mathrm{d} \boldsymbol{q}^{\prime} \mathrm{d} \boldsymbol{p}^{\prime},
$$

and this is the same with the left hand side in Eq. (22). We have used the fact, for functions $f \in \mathbb{R}$ and $G$,

$$
\iint \delta\left(f-f^{\prime}\right) G\left(f^{\prime}\right) \mathrm{d} \boldsymbol{q}^{\prime} \mathrm{d} \boldsymbol{p}^{\prime}=G(f) \iint \delta\left(f-f^{\prime}\right) \mathrm{d} \boldsymbol{q}^{\prime} \mathrm{d} \boldsymbol{p}^{\prime},
$$

where the range of $f$ is in $\mathbb{R}$ and $\iint \delta\left(f-f^{\prime}\right) \mathrm{d} \boldsymbol{q}^{\prime} \mathrm{d} \boldsymbol{p}^{\prime}<\infty$. 


\section{B Derivation of Eq. (18)}

The Eq. (18) is shown as follows: The spatial density perturbation is

$$
\delta \rho(\boldsymbol{q})=\int \delta f(\boldsymbol{q}, \boldsymbol{p}) \mathrm{d} \boldsymbol{p}=\sum_{i \in \mathbb{l}} a_{i} d_{i}(\boldsymbol{q}) .
$$

We firstly show that

$$
\iint \delta f^{*} V[\delta f] \mathrm{d} \boldsymbol{q} \mathrm{d} \boldsymbol{p}=\int \delta \rho^{*}(V * \delta \rho) \mathrm{d} \boldsymbol{q}=\sum_{i \in \mathbb{D}^{\prime}} a_{i} \int \delta \rho^{*} u_{i} \mathrm{~d} \boldsymbol{q}=\sum_{i \in \mathbb{\square}^{\prime}} \lambda_{i}\left|a_{i}\right|^{2}
$$

It is also shown that

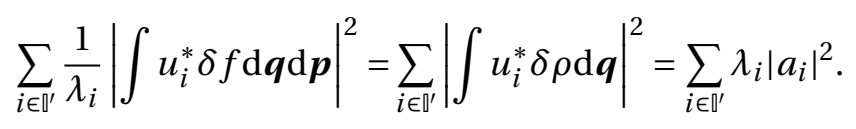

We therefore have Eq. (18).

\section{References}

[1] R. Balescu, Statistical Dynamics Matter Out of Equilibrium (Imperial College Press, 1997).

[2] A. Campa, T. Dauxois, D. Fanelli, and S. Ruffo, Physics of Long-Range Interacting Systems, (Oxford University Press, Oxford, 2014).

[3] A. Campa, T. Dauxois, and S. Ruffo, Phys. Rep. 480, 57 (2009).

[4] S. Ichimaru, Basic principles of plasmas physics a statistical approach (Westview Press, 1973).

[5] J. Binney and S. Tremaine, Galactic dynamics, 2nd ed. (Princeton University Press, Princeton, NJ, 2008).

[6] W. Braun and K. Hepp, Commun. Math. Phys. 56, 101 (1977).

[7] R. L. Dobrushin, Funct. Anal. Appl. 13, 115 (1979).

[8] H. Spohn, Large scale dynamics of interacting particles, (Springer-Verlag, Heidelberg, 1991).

[9] Y. Y. Yamaguchi, J. Barré, F. Bouchet, T. Dauxois, and S. Ruffo, Physica A 337, 36 (2004).

[10] J. Barré, F. Bouchet, T. Dauxois, S. Ruffo, and Y. Y. Yamaguchi, Physica A 365, 177 (2006).

[11] E. Caglioti and F. Rousset, J. Stat. Phys. 129, 241 (2007).

[12] E. Caglioti and F. Rousset, Arch. Rational Mech. Anal. 190, 517 (2008).

[13] M. Antoni and S. Ruffo, Phys. Rev. E 52, 2361 (1995).

[14] S. Ogawa, A. Patelli, and Y. Y. Yamaguchi, Phys. Rev. E 89, 032131 (2014).

[15] T. M. Rocha Filho, A. E. Santana, M. A. Amato, and A. Figueiredo, Phys. Rev. E 90, 032133 (2014).

[16] C. Anteneodo and C. Tsallis, Phys. Rev. Lett. 80, 5313 (1998).

[17] R. Bachelard,T. Dauxois, G. De Ninno,S. Ruffo,and F. Staniscia, Phys. Rev. E 83, 061132 (2011).

[18] A. Turchi, D. Fanelli, and X. Leoncini, Commun. Nonlinear Sci. Numeric. Sim. 16, 4718 (2011). 
[19] O. Penrose, Phys. Fluids 3, 258 (1960).

[20] S. Inagaki and T. Konishi, gravitating systems, Publ. Astron. Soc. Jpn. 45, 733 (1993).

[21] D. del-Castillo-Negrete, Phys. Plasmas, 5, 3886 (1998).

[22] P. H. Chavanis and L. Delfini, Eur. Phys. J. B 69, 389 (2009).

[23] C. Mouhot and C. Villani, J. Math. Phys, 51, 015204 (2010); Acta Math. 207, 29 (2011).

[24] V. A. Antonov, Astr. Zh. 37, 918 (1960), translated in Sov. astron. 4, 859 (1961).

[25] D. Lynden-Bell, Mon. Not. R. astr. Soc. |bf 144, 189 (1969).

[26] H. E. Kandrup, Astrophys. J. 351, 104 (1990).

[27] H. E. Kandrup, Astrophys. J. 370, 312 (1991).

[28] S. Ogawa, Phys. Rev. E 87, 062107 (2013).

[29] A. Campa and P. H. Chavanis, J. Stat. Mech, P06001 (2010).

[30] M. Lemou, F. Méhats, and P. Raphaël, Invent. Math. 187, 145 (2012).

[31] M. Lemou, A. M. Luz, and F. Méhats, Arch. Rational Mech. Anal. 224, 353 (2017).

[32] D. D. Holm, J. E. Marsden, T. Ratiu, and A. Weinstein, Phys. Rep. 123, 1 (1985).

[33] V. I. Arnold, Ann. Inst. Fourier 16, 319 (1966); Mathematical methods of classical mechanics, Second edition, (Springer, 1989).

[34] R. S. Ellis, K. Haven, and B. Turkington, Nonlinearity 15, 239 (2002).

[35] A. Patelli and S. Ruffo, Eur. Phys. J. D 68, 329 (2014).

[36] S. Ogawa, Phys. Rev. E 96, 012112 (2017).

[37] A. J. Kalnajs, Astrophys. J. 166, 275 (1971).

[38] S. Ogawa and Y. Y. Yamaguchi, Phys. Rev. E 85, 061115, (2012).

[39] M. Antoni and A. Torcini, Phys. Rev. E 57, R6233 (1998).

[40] A. Torcini and M. Antoni, Phys. Rev. E 59, 2746 (1999).

[41] J. Barré, A. Olivetti, and Y. Y. Yamaguchi, J. Stat. Mech. (2010) P08002.

[42] J. Barré, A. Olivetti, and Y. Y. Yamaguchi, J. Phys. A: Math. Theor. 44405502 (2011).

[43] J. Barré and Y. Y. Yamaguchi, J. Phys. A: Math. Theor. 46, 225501 (2013). 